\title{
Limitations of the Double-Bounce Power Estimation in Model- Based Polarimetric Decompositions of Vegetated Terrain
}

This paper was downloaded from TechRxiv (https://www.techrxiv.org).

LICENSE

CC BY 4.0

SUBMISSION DATE / POSTED DATE

$13-10-2021 / 18-10-2021$

\section{CITATION}

Watanabe, Takuma; Yamada, Hiroyoshi (2021): Limitations of the Double-Bounce Power Estimation in ModelBased Polarimetric Decompositions of Vegetated Terrain. TechRxiv. Preprint. https://doi.org/10.36227/techrxiv.16802392.v1

$\mathrm{DOI}$

10.36227/techrxiv.16802392.v1 


\title{
Limitations of the Double-Bounce Power Estimation in Model-Based Polarimetric Decompositions of Vegetated Terrain
}

\author{
Takuma Watanabe and Hiroyoshi Yamada, Member, IEEE
}

\begin{abstract}
In this research, we discuss the possibility of incorrect prediction of the double-bounce scattering (DBS) power in model-based decomposition (MBD) algorithms applied to polarimetric synthetic aperture radar (SAR) images of vegetated terrain. In most of the MBD schemes, the estimation of the DBS component is based on the assumption that the co-polarized phase difference (CPD) of the DBS is similar to those of backscattering from a pair of orthogonal planer conducting surfaces. However, for dielectric surfaces such as soil or vegetation trunks, this assumption is only valid within a certain range of radar incidence angle, which is dictated by the Brewster angles of the dielectric surfaces. If the incidence angle is out of this range, the DBS contribution is incorrectly estimated as the surface scattering. Moreover, because the Brewster angle is a function of surface permittivity, the angular range depends on moisture contents of the surfaces; therefore, correctness of the MBD results also depend on the surface moisture contents. To demonstrate this problem, we provide a simple numerical model of vegetated terrain, and we validate theoretical results by a series of controlled experiments carried out in an anechoic chamber with a simplified vegetation model.
\end{abstract}

Index Terms-synthetic aperture radar, polarimetry, modelbased decomposition, double-bounce scattering.

\section{INTRODUCTION}

$\mathbf{P}$ OLARIMETRIC synthetic aperture radar (SAR) has been widely studied in several areas of geoscience and remote sensing applications, because of its capability of rapidly providing high-resolution spatial images of the terrain in allweather and day-and-night conditions. To retrieve useful information from the polarimetric SAR images, many algorithms called target decomposition theorems have been developed and investigated until today [1]-[4]. The target decomposition theorems are broadly classified into two categories: eigenvector decomposition algorithms [5]-[8] and model-based decomposition (MBD) algorithms [9]-[22]; in this study, we focus on the latter category.

The eigenvector decomposition was originally proposed by Cloude and Pottier [6]. In this algorithm, we consider eigenvectors and eigenvalues of coherency or covariance matrices formed by the measured polarimetric scattering vectors. One of the advantages of this approach is that it provides

Manuscript received $\mathrm{xxxxx} \mathrm{xx}, \mathrm{xxxx}$; revised $\mathrm{xxxxx} \mathrm{xx}$, $\mathrm{xxxx}$; accepted xxxx xx xxxx. (Corresponding author: Takuma Watanabe.)

T. Watanabe is currently with Fujitsu Ltd., Kawasaki 2118588, Japan (email: takuma.watanabe.jp@ieee.org).

H. Yamada is with Niigata University, Niigata 9502181, Japan.

Color versions of one or more of the figures in this paper are available online at http://ieeexplore.ieee.org.

Digital Object Identifier xx.Xxxx/TGRS.xxxx.Xxxxxxx mathematically unique decomposition results. However, some approximations have to be made for physical interpretation of the decomposed scattering mechanisms [5].

The first MBD was introduced by Freeman and Durden [11], which is referred to as the Freeman-Durden decomposition (FDD). In this algorithm, the measured covariance matrices are expressed in terms of the three scattering contributions: surface scattering from the ground, volume scattering from vegetation, and double-bounce scattering (DBS) between the ground and vegetation, which is of main consideration in this study. The major advantage of the MBD is that it provides clear physical interpretation, because the covariance matrices are expanded in the known physical scattering mechanisms. However, as pointed out by several researches [10]-[13], [15][21], the FDD often produces negative power results which are physically unacceptable. This implies that the model used in the FDD does not well fit the measured covariance matrices.

To overcome this problem, many researchers have tried to extend the conventional FDD in which only a single volume scattering model was adopted; that is, the cross-polarized component in the covariance matrix was directly assigned as the volume scattering contribution, based on the assumption that the vegetation can be modeled by a cloud of randomly oriented dipoles [9]. Therefore, efforts have been made to amend the volume scattering model such that the algorithms accommodate multiple volume scattering models to describe different types of vegetated terrain. For example, Yamaguchi et al. proposed a MBD algorithm that include three different volume scattering models [10], [12]; van Zyl et al. and Arii et al. developed an adaptive volume scattering model which can represent various types of vegetated terrain, by combining the eigenvector-based decomposition and the MBD to guarantee non-negative decomposed powers [3], [15], [17]; Lee proposed other different types of adaptive volume scattering model [21].

In addition to the extended volume scattering models, another commonly employed approach is to utilize the preprocessing called deorientation that minimizes the cross-polarized term in the covariance matrices [16], [18], [19]. As the crosspolarized term is directly assigned as the volume scattering component in the FDD, the deorientation processing is expected to reduce the overestimation of the volume scattering, resulting in mitigation of the negative power problem.

Although the extended algorithms have been successfully improved the volume scattering model, however, most of the MBD schemes inherit the criterion to determine the surface and the DBS contribution; that is, co-polarized phase dif- 
ference (CPD) has been used as was originally suggested by Freeman and Durden [9]. In the original FDD, there are four equations with five unknowns. To reduce the number of unknowns, the CPD is used to determine which of the surface or the DBS components is dominant, after the subtraction of the volume scattering contribution. If the CPD is closer to $\pi$ than zero, we assume that the DBS contribution dominates and the DBS power is explicitly solved; otherwise, the surface scattering power is explicitly solved. This criterion is based on the scattering from a pair of orthogonal flat conducting surfaces, e.g., a dihedral reflector.

However, as pointed out in [6], [25], the CPD of a dihedral reflector is not always guaranteed to be $\pi$ when the reflector is made of dielectric material. This means that one cannot discriminate the surface scattering and the DBS based on the CPD, and care must be taken when we utilize the MBD. Keeping this problem in mind, the objective of this study is to demonstrate the possibility that the MBD algorithms could provide incorrect DBS and surface scattering components when the algorithms are applied to the polarimetric SAR images of vegetated terrain. The scope of the decomposition algorithms that are subjected to investigation is the MBD schemes using the CPD to determine DBS and surface scattering contributions, which include the FDD and other extended algorithms of the FDD. The main novelty and contribution of this study are to show the problem of incorrect estimation of the DBS component by a series of controlled experiments carried out in an anechoic chamber.

The remainder of this paper is organized as follows: In Section II, we discuss the two simple DBS models; that is, a dihedral reflector model and a vegetation model consisted with a single cylinder on a slightly rough ground surface. Based on these models, we show that the assumption of the CPD for estimation of the DBS power fails in certain situations. In Section III, we first review the FDD, and then apply the FDD to the data obtained from the numerical model developed in Section II to demonstrate the incorrect DBS estimation. Section IV shows the experimental validation carried out in a anechoic chamber. We experimentally prove that the incorrect DBS estimation certainly occurs in the real polarimetric SAR images. Finally, Section V concludes the study.

\section{Double-Bounce ScAttering Models}

In this section, we discuss theoretical background of the incorrect decomposition, by using simple DBS models. For this aim, we construct a simple backscattering model of vegetated terrain.

\section{A. Notations}

First, we describe some symbols and notations used in this paper. We designate a polarization channel corresponding to $p$ polarized transmission and $q$-polarized reception as $q p$, where $p, q \in\{h, v\}$ and $h$ and $v$ represent horizontal and vertical polarization states, respectively. We define the scattering matrix of a scatterer as

$$
\boldsymbol{S}_{\xi}=\left[\begin{array}{ll}
S_{h h}^{\xi} & S_{h v}^{\xi} \\
S_{v h}^{\xi} & S_{v v}^{\xi}
\end{array}\right]=\left[\begin{array}{ll}
S_{h h}^{\xi} & S_{h v}^{\xi} \\
S_{h v}^{\xi} & S_{v v}^{\xi}
\end{array}\right]
$$

TABLE I

COEFFICIENTS OF THE SOIL PERMITTIVITY MODEL AT $10 \mathrm{GHz}$ [23].

\begin{tabular}{c|c|c|c}
\hline$n$ & 0 & 1 & 2 \\
\hline$a_{n}$ & $2.502-j 0.070$ & $-0.003+j 0.000$ & $-0.003+j 0.001$ \\
\hline$b_{n}$ & $10.101+j 6.620$ & $0.221+j 0.015$ & $-0.004-j 0.081$ \\
\hline$c_{n}$ & $77.482+j 21.578$ & $-0.061+j 0.293$ & $-0.135+j 0.332$ \\
\hline
\end{tabular}

where $\xi \in\{\perp, R, g, v, g c, c g, d, t, m\}$ refers to a scattering mechanism associated to this scattering matrix, which is described later.

Moreover, we define the corresponding scattering vector $\boldsymbol{s}_{\xi}$ and the covariance matrix $\boldsymbol{C}_{\xi}$ as

$$
\begin{aligned}
\boldsymbol{s}_{\xi}= & {\left[\begin{array}{lll}
S_{h h}^{\xi} & \sqrt{2} S_{h v}^{\xi} & S_{v v}^{\xi}
\end{array}\right]^{T} } \\
\boldsymbol{C}_{\xi}= & {\left[\begin{array}{lll}
C_{h h h h}^{\xi} & C_{h h h v}^{\xi} & C_{h h v v}^{\xi} \\
C_{h v h h}^{\xi} & C_{h v h v}^{\xi} & C_{h v v v}^{\xi} \\
C_{v v h h}^{\xi} & C_{v v h v}^{\xi} & C_{v v v v}^{\xi}
\end{array}\right]=\boldsymbol{s}_{\xi} \boldsymbol{s}_{\xi}^{\dagger} } \\
= & {\left[\begin{array}{ccc}
S_{h h}^{\xi} S_{h h}^{\xi *} & \sqrt{2} S_{h h}^{\xi} S_{h v}^{\xi *} & S_{h h}^{\xi} S_{v v}^{\xi *} \\
\sqrt{2} S_{h v}^{\xi} S_{h h}^{\xi *} & 2 S_{h v}^{\xi} S_{h v}^{\xi *} & \sqrt{2} S_{h v}^{\xi} S_{v v}^{\xi *} \\
S_{v v}^{\xi} S_{h h}^{\xi *} & \sqrt{2} S_{v v}^{\xi} S_{h v}^{\xi *} & S_{v v}^{\xi} S_{v v}^{\xi *}
\end{array}\right] }
\end{aligned}
$$

where $[\cdot]^{T},[\cdot]^{\dagger}$ and $[\cdot]^{*}$ represent the transpose, the conjugate transpose, and the complex conjugate operations, respectively. If multiple scatterers are considered, the covariance matrices must be averaged and that matrix is written as $\left\langle\boldsymbol{C}_{\xi}\right\rangle$, where

$\langle\cdot\rangle$ represents ensemble averaging, and the elements of this matrix is denoted as $\left\langle C_{q p s r}^{\xi}\right\rangle$. Using the covariance matrix of Eq. (3), we can calculate the backscattering coefficient $\sigma_{q p s r}^{\xi}$, the co-polarized backscattering coefficient $\sigma_{q p}^{\xi}$, and the phase difference $\phi_{q p s r}^{\xi}$ as

$$
\begin{aligned}
\sigma_{q p s r}^{\xi} & =4 \pi\left|C_{q p s r}^{\xi}\right| \\
\sigma_{q p}^{\xi} & =\sigma_{q p q p}^{\xi} \\
\phi_{q p s r}^{\xi} & =\arg C_{q p s r}^{\xi}=\arg S_{q p}^{\xi}-\arg S_{s r}^{\xi}
\end{aligned}
$$

where $r, s \in\{h, v\}$. The CPD can be computed from Eq. (4c) with $q p \rightarrow h h$ and $s r \rightarrow v v$.

\section{B. Permittivity Models of Vegetated Terrain}

Before developing DBS models, we summarize permittivity models for soil and vegetation because the DBS models require these quantity as model input parameters.

1) Soil: Several permittivity models for soil exist in literature. In this study, we employ the model proposed by Hallikainen et al. [23], which is given by the following polynomial expression:

$$
\begin{aligned}
\varepsilon_{r}^{g}=\left(a_{0}+a_{1} Q_{s}+a_{2} Q_{c}\right) & +\left(b_{0}+b_{1} Q_{s}+b_{2} Q_{c}\right) m_{g} \\
& +\left(c_{0}+c_{1} Q_{s}+c_{2} Q_{c}\right) m_{g}^{2}
\end{aligned}
$$

where $Q_{s}$ and $Q_{c}$ are the content rates of sand and clay, respectively. The coefficients $a_{n}, b_{n}$, and $c_{n}(n \in\{0,1,2\})$ in Eq. (5) are given in [23] and reproduced in Table I for $10 \mathrm{GHz}$. 


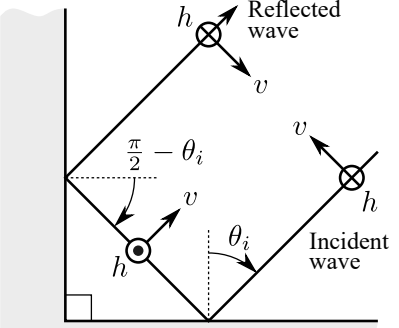

Perfect electric conductor

(a)

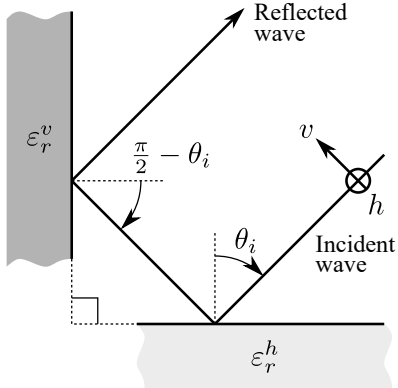

(b)
Fig. 1. Geometry of dihedral reflectors made of different materials. The phase flipping of the $v$-polarized wave is not necessarily guaranteed for the dielectric case. (a) Conducting surfaces. (b) Dielectric surfaces.

2) Vegetation: For vegetation, we employ the following permittivity model proposed by Ulaby and El-rayes [24].

$$
\varepsilon_{r}^{v}=\varepsilon_{n}+v_{f} \varepsilon_{f}+v_{b} \varepsilon_{b}
$$

where $\varepsilon_{n}, \varepsilon_{f}$, and $\varepsilon_{b}$ represent the nondispersive residual part of the dielectric constant, the dielectric constant of free water, and the dielectric constant of bound water, respectively; the expressions for them are given as follows:

$$
\begin{aligned}
& \varepsilon_{n}=1.7+3.2 m_{v}+6.5 m_{v}^{2} \\
& \varepsilon_{f}=4.9+\frac{75}{1+j f_{\mathrm{GHz}} / 18}-j \frac{18 \sigma_{s}}{f_{\mathrm{GHz}}} \\
& \varepsilon_{b}=2.9+\frac{55}{1+\sqrt{j f_{\mathrm{GHz}} / 0.18}}
\end{aligned}
$$

where $m_{v}$ is the volumetric water content, $f_{\mathrm{GHz}}$ is the frequency in $\mathrm{GHz}$, and $\sigma_{s}$ is a function of a salinity $Q_{v}$ of the water, which is defined as

$$
\sigma_{s}=0.16 Q_{v}-0.0013 Q_{v}^{2}
$$

In Eq. (6), $v_{f}$ and $v_{b}$ are the volume fraction of free water and the volume fraction of vegetation-bound water, respectively; they are given by the following expressions:

$$
\begin{aligned}
v_{f} & =m_{v}\left(0.82 m_{v}+0.166\right) \\
v_{b} & =\frac{31.4 m_{v}^{2}}{1+59.5 m_{v}^{2}}
\end{aligned}
$$

\section{A Pair of Orthogonal Surfaces}

As the simplest DBS model, we consider backscattering from a pair of orthogonal flat surfaces, that is, a dihedral reflector shown in Fig. 1(a). If the surfaces are made of conducting material, the scattering matrix $\boldsymbol{S}_{\perp}$ is given by

$$
\boldsymbol{S}_{\perp} \propto\left[\begin{array}{cc}
1 & 0 \\
0 & -1
\end{array}\right] .
$$

The CPD $\phi_{h h v v}^{\perp}$ corresponding to Eq. (10) is

$$
\phi_{h h v v}^{\perp}=\pi \text {. }
$$

Therefore, the CPD of $\pi$ indicates existence of a scatterer which causes the dihedral-like DBS. This is the basic assumption used in most of the MBD algorithms.
However, situation is not as simple as the case of a conducting dihedral scatterer when the material is replaced with dielectric one. Next, let us consider this case. When a plane wave is incident on a smooth flat surface with relative permittivity $\varepsilon_{r}$, we denote the reflection coefficients for $h$ - and $v$-polarized waves as $R_{h}\left(\varepsilon_{r}, \theta_{i}\right)$ and $R_{v}\left(\varepsilon_{r}, \theta_{i}\right)$, respectively, where $\theta_{i}$ represents the incidence angle measured from a normal vector of this surface. These reflection coefficients are known as the Fresnel coefficients and given by the following expressions:

$$
\begin{aligned}
& R_{h}\left(\varepsilon_{r}, \theta\right)=\frac{\cos \theta-\sqrt{\varepsilon_{r}-\sin ^{2} \theta}}{\cos \theta+\sqrt{\varepsilon_{r}-\sin ^{2} \theta}} \\
& R_{v}\left(\varepsilon_{r}, \theta\right)=\frac{\varepsilon_{r} \cos \theta-\sqrt{\varepsilon_{r}-\sin ^{2} \theta}}{\varepsilon_{r} \cos \theta+\sqrt{\varepsilon_{r}-\sin ^{2} \theta}}
\end{aligned}
$$

The Fresnel coefficients of Eq. (12) describe the reflectivity of specular reflection. If the incidence angle $\theta_{i}$ is

$$
\theta_{i}=\theta_{B}=\tan ^{-1}\left(\sqrt{\varepsilon_{r}}\right),
$$

the vertically polarized Fresnel coefficient $R_{v}\left(\varepsilon_{r}, \theta\right)$ becomes zero. This angle $\theta_{B}$ is known as the Brewster angle or the polarization angle.

Based on Eq. (12), we consider the backscattering of a pair of orthogonal dielectric surfaces, as depicted in Fig. 1(b), where the relative permittivity of the horizontal and the vertical surfaces are $\varepsilon_{r}^{h}$ and $\varepsilon_{r}^{v}$, respectively. In this case, the reflection coefficients for the horizontally and the vertically polarized waves can be given by

$$
\begin{aligned}
R_{h h} & =R_{h}\left(\varepsilon_{r}^{h}, \theta_{i}\right) R_{h}\left(\varepsilon_{r}^{v}, \pi / 2-\theta_{i}\right) \\
R_{v v} & =-R_{v}\left(\varepsilon_{r}^{h}, \theta_{i}\right) R_{v}\left(\varepsilon_{r}^{v}, \pi / 2-\theta_{i}\right) .
\end{aligned}
$$

Thus, the scattering matrix for this case is

$$
\boldsymbol{S}_{R} \propto\left[\begin{array}{cc}
R_{h h} & 0 \\
0 & R_{v v}
\end{array}\right] .
$$

The backscattering coefficients and the CPD corresponding to Eq. (15) can be given by Eq. (4a) and Eq. (4c) with $\xi \rightarrow R$.

In this case, according to Eq. (13), the following two Brewster angles for the horizontal and the vertical surfaces exist, denoted by $\theta_{B}^{h}$ and $\theta_{B}^{v}$, respectively:

$$
\begin{aligned}
& \theta_{B}^{h}=\tan ^{-1}\left(\sqrt{\varepsilon_{r}^{h}}\right) \\
& \theta_{B}^{v}=\pi / 2-\tan ^{-1}\left(\sqrt{\varepsilon_{r}^{v}}\right)
\end{aligned}
$$

If the incidence angle approaches to the Brewster angles given by Eq. (16), the backscattering coefficient is expected to be significantly reduced. In [25], this phenomenon was confirmed for the polarimetric SAR images obtained from a laboratory experiment; in [26], similar observation was made for manmade targets in TerraSAR-X images.

As an example, we computed the Fresnel reflection coefficients for the backscattering case defined in Eq. (14). The dielectric constants were determined using Eq. (5) for $\varepsilon_{r}^{h}$ and Eq. (6) for $\varepsilon_{r}^{v}$, by setting the sand content to $Q_{s}=0.5(50 \%)$, the clay content to $Q_{c}=0.15(15 \%)$, and vegetation salinity to $Q_{v}=8.5 \mathrm{~g} / \mathrm{kg}$. For simplicity, the moisture contents of 


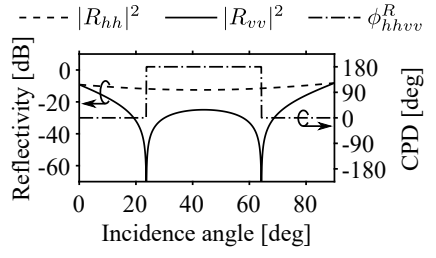

(a)



(b)
Fig. 2. Fresnel reflection coefficient for DBS. The dielectric constants were taken from the models given in Section II-B, ignoring the imaginary parts for simplicity of the discussion. (a) Dry condition $\left(m_{g}=m_{v}=0.1\right)$. (b) Wet condition $\left(m_{g}=m_{v}=0.5\right)$

the soil and the vegetation were set to be equal, and only the real parts of the derived complex permittivities were retained. Two moisture contents were considered, namely, $m_{g}=m_{v}=0.1(10 \%)$ and $m_{g}=m_{v}=0.5(50 \%)$, where the former and the latter were referred to as the dry and the wet conditions, respectively. For these cases, the determined relative permittivities were $\varepsilon_{r}^{h} \approx 4.3$ and $\varepsilon_{r}^{v} \approx 5.2$ for the dry condition, and $\varepsilon_{r}^{h} \approx 30.0$ and $\varepsilon_{r}^{v} \approx 26.8$ for the wet condition. The corresponding Brewster angles were $\theta_{B}^{h}=64.2^{\circ}$ and $\theta_{B}^{v}=23.7^{\circ}$ for the dry condition, and $\theta_{B}^{h}=79.1^{\circ}$ and $\theta_{B}^{v}=10.9^{\circ}$ for the wet condition.

The magnitude $\left|R_{p p}\right|^{2}$ and the CPD are displayed in Fig. 2(a) and (b) for the dry and the wet conditions. At the Brewster angles, we can clearly recognize nulls in the reflection coefficients. As shown in Fig. 2, the phase flipping of the dihedral reflector is no longer guaranteed for these dielectric cases; the phase difference $\pi$ occurs only within the range of the incidence angle between the two Brewster angles. This range depends on the moisture contents; that is, as the moisture contents get higher, the two Brewster angles leave from $45^{\circ}$.

From the abovementioned observations, we can deduce that the phase-flipping assumption of Eq. (11) possibly fails, and as a result, the DBS can be incorrectly estimated in the following situations:

1) The moisture content is constant but the observation geometry is changed such that the incidence angle exceeds the angular range between the two Brewster angles, that is, $\theta_{i} \notin\left(\theta_{B}^{v}, \theta_{B}^{g}\right)$.

2) The incidence angle is fixed but the dielectric constants are changed because of the variation of the moisture contents of a dihedral reflector, resulting $\theta_{i} \notin\left(\theta_{B}^{v}, \theta_{B}^{g}\right)$.

In the following discussion, we show that the physically incorrect results of MBD is certainly observed for vegetated terrain, by using a numerical model and an experimental model.

\section{A Cylinder on a Rough Surface}

Next, we extend our discussion to a more realistic model for vegetated terrain, in which a single dielectric cylinder with finite length stands vertically on a rough dielectric surface, as shown in Fig. 3. Although this model is still simplistic to describe general backscattering from vegetated terrain, however, the model can be readily understood and the model has a clear physical insight. Our main focus is to show the

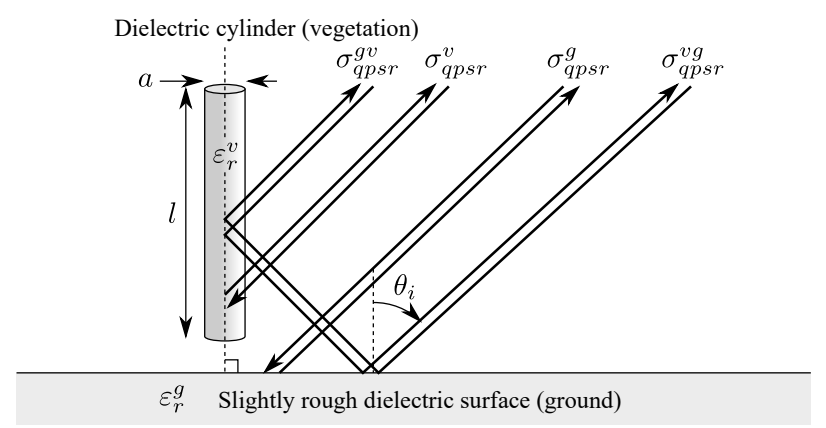

Fig. 3. Geometry of the simplified vegetation model. A single dielectric cylinder stands vertically on a slightly rough dielectric ground surface. The total backscattering is considered to be the sum of the four scattering mechanisms, namely, $\sigma_{q p s r}^{g}, \sigma_{q p s r}^{v}, \sigma_{q p s r}^{g v}$, and $\sigma_{q p s r}^{v g}$.

incorrect DBS estimation for vegetated terrain, rather than to develop an accurate vegetation scattering model; therefore, this model can be considered as an idealized theoretical model to enhance the DBS component which is expected to occur in the scattering from vegetated terrain. More complicated models including multiple vegetation components, cylinder orientation, and wave attenuation effects have been extensively studied in literature (for example, see [3], [27]).

Our modeling process follows the approach presented in [3], except that only a single vertical cylinder is considered, excluding the attenuation factor owing to the wave propagation through a layer of randomly distributed cylinders.

1) Direct Backscattering from the Ground $\left(\sigma_{q p s r}^{g}\right)$ : We use a small-perturbation model for a rough surface, as a model to describe the backscattering from the ground surface. If the exponential correlation function is considered, the scattering matrix based on the small-perturbation model is given by [3]

$$
\begin{aligned}
\boldsymbol{S}_{g} & =\left[\begin{array}{cc}
S_{h h}^{g} & S_{h v}^{g} \\
S_{v h}^{g} & S_{v v}^{g}
\end{array}\right]=\left[\begin{array}{cc}
S_{h h}^{g} & 0 \\
0 & S_{v v}^{g}
\end{array}\right] \\
S_{p p}^{g} & =k^{2} h_{g} \cos ^{2} \theta_{i} \alpha_{p p}\left(\varepsilon_{r}^{g}, \theta_{i}\right) \sqrt{W\left(2 k \sin \theta_{i}\right)}
\end{aligned}
$$

where

$$
\begin{aligned}
\alpha_{h h}\left(\varepsilon_{r}^{g}, \theta_{i}\right) & =\frac{\varepsilon_{r}^{g}-1}{\left(\cos \theta_{i}+\sqrt{\varepsilon_{r}^{g}-\sin ^{2} \theta_{i}}\right)^{2}} \\
\alpha_{v v}\left(\varepsilon_{r}^{g}, \theta_{i}\right) & =\frac{\left(\varepsilon_{r}^{g}-1\right)\left[\left(\varepsilon_{r}^{g}-1\right) \sin ^{2} \theta_{i}+\varepsilon_{r}^{g}\right]}{\left(\varepsilon_{r}^{g} \cos \theta_{i}+\sqrt{\varepsilon_{r}^{g}-\sin ^{2} \theta_{i}}\right)^{2}}
\end{aligned}
$$

with

$$
W\left(2 k \sin \theta_{i}\right)=\frac{2 l_{g}^{2}}{\pi\left[1+\left(4 k l_{g} \sin \theta_{i}\right)^{2}\right]^{3 / 2}} .
$$

The relative permittivity $\varepsilon_{r}^{g}$ is given by Eq. (5), and $h_{g}$ is the root mean square (RMS) height of the surface. The function $W\left(2 k \sin \theta_{i}\right)$ of Eq. (19), with wavenumber $k$, represents the roughness spectrum of a surface with the exponential correlation function; $l_{g}$ represents the correlation length. We define the corresponding covariance matrix $\boldsymbol{C}_{g}$ and the backscattering coefficient $\sigma_{q p s r}^{g}$ as Eq. (3) and Eq. (4a) with $\xi \rightarrow g$, respectively. 
2) Direct Backscattering from Vegetation $\left(\sigma_{q p s r}^{v}\right)$ : We consider a dielectric cylinder of finite length, which is vertically stands on the ground surface, as shown in Fig. 3. We denote the bistatic scattering matrix for this cylinder as

$$
\boldsymbol{S}_{c}\left(\theta_{i}, \theta_{s}\right)=\left[\begin{array}{ll}
S_{h h}^{c} & S_{h v}^{c} \\
S_{v h}^{c} & S_{v v}^{c}
\end{array}\right]
$$

where $\theta_{s}$ is the scattering angle; note that the backscattering corresponds to $\theta_{s}=\theta_{i}$. Several researchers have developed the expressions for the scattering from a dielectric cylinder with finite length; in this study, we employ the model proposed by Sarabandi, and the detailed expressions for $S_{q p}^{c}$ in Eq. (20) are given in [3], [28], [29].

Based on the bistatic scattering matrix of Eq. (20), the scattering matrix representing the direct backscattering from a dielectric cylinder (i.e., a vegetation trunk) is defined as

$$
\boldsymbol{S}_{v}=\boldsymbol{S}_{c}\left(\theta_{i}, \theta_{i}\right) .
$$

Moreover, the corresponding covariance matrix $\boldsymbol{C}_{v}$ and the backscattering coefficient $\sigma_{q p s r}^{v}$ for Eq. (21) are given by Eq. (3) and Eq. (4a), respectively, with $\xi \rightarrow v$.

3) Double-Bounce Scattering ( $\sigma_{q p s r}^{g v}$ and $\sigma_{q p s r}^{v g}$ ): To model the DBS between the ground and the vegetation, we first consider specular scattering for a rough ground surface [3], [27], [30], [31] given by

$$
\boldsymbol{R}_{g}=\left[\begin{array}{cc}
R_{h}\left(\varepsilon_{r}^{g}, \theta_{i}\right) & 0 \\
0 & R_{v}\left(\varepsilon_{r}^{g}, \theta_{i}\right)
\end{array}\right] e^{-2 k^{2} h_{g}^{2} / \cos ^{2} \theta_{i}}
$$

where $h_{g}$ is the RMS height of the surface.

The DBS between the ground and the vegetation comprises two scattering mechanisms, namely, scattering by the vegetation followed by scattering by the ground $\left(\boldsymbol{S}_{v g}\right)$, and the same scattering path taken place in the opposite direction $\left(\boldsymbol{S}_{g v}\right)$. The individual scattering matrices for these cases are given by

$$
\begin{aligned}
\boldsymbol{S}_{g v} & =\boldsymbol{S}_{c} \boldsymbol{J} \boldsymbol{R}_{g} \\
\boldsymbol{S}_{v g} & =\boldsymbol{R}_{g} \boldsymbol{J} \boldsymbol{S}_{c}
\end{aligned}
$$

where

$$
\boldsymbol{J}=\left[\begin{array}{cc}
-1 & 0 \\
0 & 1
\end{array}\right] \text {. }
$$

Since these scattering mechanisms take place in the same propagation paths, the total DBS can be computed via coherently summing these scattering matrices, as follows:

$$
\boldsymbol{S}_{d}=\boldsymbol{S}_{g v}+\boldsymbol{S}_{v g}
$$

where the elements of the scattering matrix $\boldsymbol{S}_{d}$ are denoted as Eq. (1) with $\xi \rightarrow d$. Moreover, the covariance matrix $\boldsymbol{C}_{d}$ and the backscattering coefficients $\sigma_{q p s r}^{d}$ for Eq. (25) are given by Eq. (3) and Eq. (4a), respectively, with $\xi \rightarrow d$.

4) Total Backscattering: Now that we have derived the covariance matrices corresponding to individual scattering mechanisms, namely, $\boldsymbol{C}_{g}, \boldsymbol{C}_{d}$, and $\boldsymbol{C}_{v}$, the final step for obtaining the total backscattering from the vegetated terrain is to compute the sum of these contributions, as follows:

$$
C_{t}=C_{g}+C_{d}+C_{v}
$$

where the elements of the covariance matrix $C_{q p s r}^{t}$ are denoted as Eq. (3), and the total backscattering $\sigma_{t}$ is given by Eq. (4a), with the appropriate replacement $\xi \rightarrow t$.
TABLE II

MODEL INPUT PARAMETERS.

\begin{tabular}{l|l}
\hline Polarization $q p$ & $h h, h v, v h, v v$ \\
Frequency & $10 \mathrm{GHz}$ (X-band) \\
Incidence angle $\theta_{i}$ & $0^{\circ}$ to $90^{\circ}$ \\
\hline Cylinder diameter $a$ & $2 \mathrm{~cm}$ \\
Cylinder length $l$ & $30 \mathrm{~cm}$ \\
Cylinder orientation & Vertical \\
Cylinder moisture content $m_{v}$ & $10 \%$ (dry), $50 \%$ (wet) \\
Cylinder salinity $Q_{v}$ & $8.5 \mathrm{~g} / \mathrm{kg}$ \\
\hline Ground correlation length $l_{g}$ & $0.1 \mathrm{~cm}$ \\
Ground RMS height $h_{g}$ & $10^{-6} \mathrm{~cm}$ \\
Soil moisture content $m_{g}$ & $10 \%$ (dry), $50 \%$ (wet) \\
Soil sand content $Q_{s}$ & $50 \%$ \\
Soil clay content $Q_{c}$ & $15 \%$ \\
\hline
\end{tabular}

5) Numerical Results and Discussion: Here, we provide numerical examples of the backscattering model for vegetated terrain, given in Eq. (26). Table II lists the input parameters for this model. Frequency of the transmitting wave was $10 \mathrm{GHz}$, and the incidence angle was varied from $0^{\circ}$ to $90^{\circ}$. Diameter and length of the cylinder were $a=2 \mathrm{~cm}$ and $l=30 \mathrm{~cm}$, respectively. These parameters were selected to ensure the consistency of the experimental validation discussed in Section IV. Correlation length and RMS height of the ground were $0.1 \mathrm{~cm}$ and $10^{-6} \mathrm{~cm}$, respectively. For simplicity, we assumed that moisture contents of the cylinder and the soil were equal such that $m_{v}=m_{g}$. We considered two different moisture content, namely, $10 \%$ and $50 \%$, where the former and the latter are referred to as the dry and the wet conditions, respectively, in the following discussion.

Fig. 4 and Fig. 5 show the calculated co-polarized backscattering coefficients and the CPD for the dry and the wet conditions, respectively. In these figures, (a) shows the individual backscattering coefficients, and (b) shows the total backscattering coefficients plotted with the CPD. Because the model had no cross-polarized contribution (i.e., $h v$ and $v h$ ), only the co-polarized components (i.e., $h h$ and $v v$ ) are displayed in these figures.

From Fig. 4(a) and Fig. 5(a), we can confirm that the DBS components (i.e., $\sigma_{h h}^{d}$ and $\sigma_{v v}^{d}$ ) are dominant for almost all the incidence angles, for both the dry and the wet conditions. Moreover, we can recognize that the $v v$-polarized backscattering coefficients have dips corresponding to the Brewster angles, whereas the variations of the $h h$-polarized backscattering coefficients are moderate with respect to the change in the incidence angle. Comparing Fig. 4 and Fig. 5 reveals that the separation of the two Brewster angle dips becomes wider as the moisture contents become larger.

Because of the dominant DBS, the total backscattering coefficients shown in Fig. 4(b) and Fig. 5(b) resemble the individual DBS components shown in 4(b) and Fig. 5(b). When we consider continuously varying the incidence angle from $0^{\circ}$ to $90^{\circ}$, the $\mathrm{CPD}$ begins to approach $\pm 180^{\circ}$ when the incidence angle is close to the first Brewster angle corresponding to the cylinder (around $15^{\circ}$ for the dry condition, and around $14^{\circ}$ for the wet condition). When the incidence angles exceed the second Brewster angle corresponding to the ground (around $65^{\circ}$ for the dry condition and around $78^{\circ}$ for 


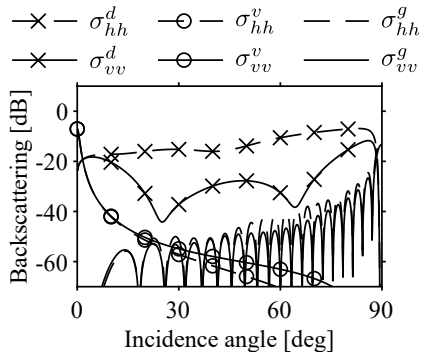

(a)

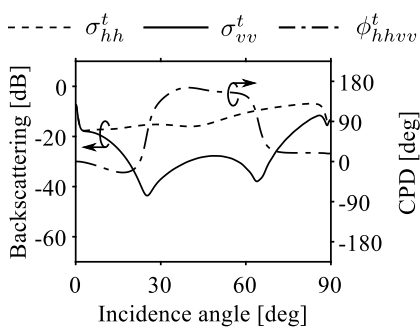

(b)
Fig. 4. Co-polarized backscattering coefficients and CPD (simulation, dry condition). (a) Individual backscattering. (b) Total backscattering and CPD.

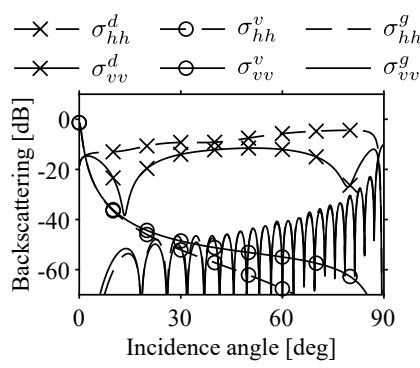

(a)

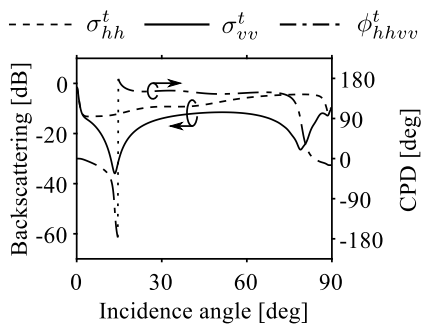

(b)
Fig. 5. Co-polarized backscattering coefficients and CPD (simulation, wet condition). (a) Individual backscattering. (b) Total backscattering and CPD.

the wet condition), the CPD goes back to near $0^{\circ}$.

As mentioned in Section II-C, most of the MBD algorithms relies on the CPD to infer the existence of double-bounce scatterers. Though this assumption is valid when the incidence angle is within the angular range between the two Brewster angles, the phase flipping is not necessarily guaranteed as we can confirm from these numerical examples. In Section III-C, we apply the FDD to the data obtained from the numerical model to show that the incorrect results are certainly produced owing to the problem discussed so far.

\section{Model-BASEd Polarimetric DeCOMPosition}

In this section, we first provide a short review of the original FDD. Then, based on the DBS model given in Section II, we examine behavior of the FDD in terms of the power estimation of the DBS to show the physically incorrect result.

\section{A. Freeman-Durden Decomposition}

In the framework of the FDD, we consider the decomposition of the measured covariance matrix $\left\langle\boldsymbol{C}_{m}\right\rangle$ into a sum of the three scattering contributions, namely, the direct backscattering from the ground $\left(\overline{\boldsymbol{C}}_{g}\right)$, the double-bounce interaction between the ground and the vegetation trunk $\left(\overline{\boldsymbol{C}}_{d}\right)$, and the direct backscattering from randomly oriented branches $\left(\overline{\boldsymbol{C}}_{v}\right)$, as follows [9]:

$$
\left\langle\boldsymbol{C}_{m}\right\rangle=f_{s} \overline{\boldsymbol{C}}_{g}+f_{d} \overline{\boldsymbol{C}}_{d}+f_{v} \overline{\boldsymbol{C}}_{v}
$$

where $\overline{\boldsymbol{C}}_{g}, \overline{\boldsymbol{C}}_{d}$, and $\overline{\boldsymbol{C}}_{v}$ are defined as follows:

$$
\overline{\boldsymbol{C}}_{g}=\left[\begin{array}{ccc}
|\beta|^{2} & 0 & \beta \\
0 & 0 & 0 \\
\beta^{*} & 0 & 1
\end{array}\right], \quad \overline{\boldsymbol{C}}_{d}=\left[\begin{array}{ccc}
|\alpha|^{2} & 0 & \alpha \\
0 & 0 & 0 \\
\alpha^{*} & 0 & 1
\end{array}\right],
$$

$$
\overline{\boldsymbol{C}}_{v}=\left[\begin{array}{ccc}
|\beta|^{2} & 0 & 1 / 3 \\
0 & 2 / 3 & 0 \\
1 / 3 & 0 & 1
\end{array}\right] .
$$

According to this model, we have the following four equations with five unknowns.

$$
\begin{aligned}
& \left\langle C_{h h h h}^{m}\right\rangle=\left\langle\left|S_{h h}^{m}\right|^{2}\right\rangle=f_{s}|\beta|^{2}+f_{d}|\alpha|^{2}+f_{v} \\
& \left\langle C_{v v v v}^{m}\right\rangle=\left\langle\left|S_{v v}^{m}\right|^{2}\right\rangle=f_{s}+f_{d}+f_{v} \\
& \left\langle C_{h v h v}^{m}\right\rangle=\left\langle\left|S_{h v}^{m}\right|^{2}\right\rangle=f_{v} / 3 \\
& \left\langle C_{h h v v}^{m}\right\rangle=\left\langle S_{h h}^{m} S_{v v}^{m *}\right\rangle=f_{s} \beta+f_{d} \alpha+f_{v} / 3
\end{aligned}
$$

As mentioned in Section II-C, Freeman and Durden suggested the use of the CPD to determine the unknown constants $\alpha$ and $\beta$ in Eq. (29) [9]. If we are observing a conducting dihedral reflector, the CPD $\phi_{h h v v}$ is expected to be $\pi$ as given in Eq. (11). Therefore, if the CPD is closer to $\pi$ than zero, we assume that the DBS contribution dominates and the constant $\alpha$ is explicitly solved by setting the constant $\beta=1$. Otherwise, we explicitly solve $\beta$ by setting $\alpha=-1$, assuming that the surface scattering contribution is dominant. This determination is done via the following criteria:

$$
\begin{cases}\alpha=-1 & \text { if } \Re\left\langle S_{h h}^{m} S_{v v}^{m *}\right\rangle \geq 0 \\ \beta=1 & \text { if } \Re\left\langle S_{h h}^{m} S_{v v}^{m *}\right\rangle<0\end{cases}
$$

where $\Re$ represents the real part. The criteria in Eq. (30) can also be expressed in terms of the CPD $\phi_{h h v v}^{m}$ as follows:

$$
\left\{\begin{array}{ll}
\alpha=-1 & \text { if } \phi_{h h v v}^{m} \in[-\pi / 2, \pi / 2] \\
\beta=1 & \text { otherwise }
\end{array} .\right.
$$

However, this criterion is inappropriate when the incidence angle of the observation is outside of the angular range between two Brewster angles, as we already exemplified using the numerical model of the scattering from vegetated terrain. In Section III-C, we confirm this phenomenon by applying the FDD to the numerical model given by Eq. (26).

\section{B. Other Extended Algorithms}

Since the introduction of the original FDD, many extended versions have been proposed [10]-[22], as discussed in Section I. These advanced MBD algorithms mainly consider the extension of the volume scattering model to deal with various types of vegetated terrain. For example, Yamaguchi et al. included the fourth component representing helix scattering, and considered three different volume scattering models, namely, uniform random distribution, cosine-squared distributions around vertical direction, and that around horizontal direction [10]. However, after subtracting the volume and the helix contributions, the DBS and the surface scattering are determined by the same process as in the original FDD. The MBD proposed by van Zyl et al. [17] and Arii et al. [15] can directly provide the parameters $\beta$ and $\alpha$, based on non-negative eigenvalue constraint. The artificial assignment defined in Eq. (31) can be avoided, however, the CPD is still used to determine which of the DBS or surface scattering contributions is dominant. Therefore, these algorithms and other extended algorithms using similar CPD criterion inherently suffer the problem of incorrect estimation of the DBS contribution, as discussed in Section III-A. 




(a)

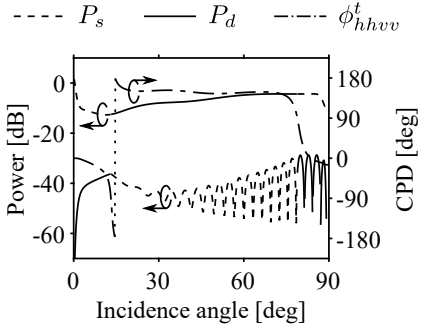

(b)
Fig. 6. FDD results (simulation). We tested Y40 and AMBD and confirmed that the similar results were obtained. (a) Dry condition. (b) Wet condition.

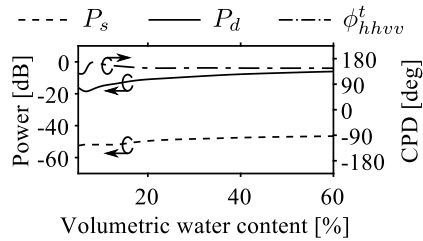

(a)

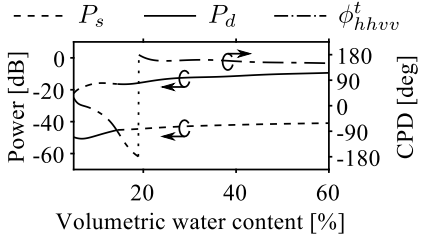

(b)
Fig. 7. FDD results at different incidence angles with respect to the volumetric moisture content (simulation). We tested Y40 and AMBD and confirmed that the similar results were obtained. (a) $\theta_{i}=45^{\circ}$. (b) $\theta_{i}=20^{\circ}$.

\section{Decomposed Results}

We applied the FDD to the theoretical backscattering model for vegetated terrain developed in Section II-D. Fig. 6(a) and (b) show the decomposed power of FDD applied to the numerical model in the dry (i.e., $m_{v}=m_{g}=10 \%$ ) and the wet (i.e., $m_{v}=m_{g}=50 \%$ ) conditions, respectively, plotted with the CPD $\phi_{h h v v}^{t}$. Note that we also tested the Yamaguchi decomposition (Y40) [10] and the adaptive modelbased decomposition (AMBD) proposed by Arii et al. [3], [15], and confirmed that the similar results were obtained. In Fig. 6, $P_{s}=\left|f_{s}\right|^{2}$ and $P_{d}=\left|f_{d}\right|^{2}$ represent the surface and the DBS power, respectively. Since no cross-polarized backscattering exited in the numerical model, no volume scattering power was observed.

As expected, the DBS component is dominant at the incidence angles between the two Brewster angles. However, as the CPD approaches to $0^{\circ}$, the surface scattering is greater than the DBS, though the ground-cylinder double-bounce interaction must be dominant in our numerical model, as shown in Fig. 4 and Fig. 5. These physically incorrect results can be readily understood from the CPD criterion of Eq. (31) which is used in the FDD and other extended algorithms.

Next, we consider fixing the incidence angle while varying the moisture contents of the ground and the cylinder. The other model input parameters were same as those listed in Table II. Note that the ground and the cylinder moisture contents were simultaneously varied by setting them equal $\left(m_{g}=m_{v}\right)$. Fig. 7(a) and (b) show the decomposed power of the FDD at the incidence angle $45^{\circ}$ and $20^{\circ}$, respectively, plotted with the $\mathrm{CPD} \phi_{h h v v}^{t}$. In Fig. 7, we varied the volumetric water content from $5 \%$ to $60 \%$.

As one can see from Fig. 7(a), the decomposed power is dominated by the DBS, because the CPD is always greater than $90^{\circ}$. This is a physically reasonable result since the groundcylinder double-bounce interaction was dominant, as shown in Fig. 4(a). In contrast, different trend was obtained when the incidence angle was changed to $\theta_{i}=20^{\circ}$. As shown in Fig. 4(b), the decomposed power is dominated by the surface scattering component when the volumetric water content is less than about $15 \%$, which is a physically unacceptable result. For the moisture content greater than $15 \%$, the DBS component is again dominant, as in Fig. 7(a).

This phenomenon can be understood from the CPD in Fig. 7(b). At the smallest moisture content $\left(m_{g}=m_{v}=5 \%\right)$, the CPD is closer to zero than $\pi$, resulting the dominant surface component. The CPD $\phi_{h h v v}^{m}$ gradually approaches $-180^{\circ}$ with the increase in the volumetric water content. This is because that the first Brewster angle corresponding to the cylinder moves away from $45^{\circ}$ for a larger moisture content. When the Brewster angle dip was about to cross over the incidence angle, the CPD exceeded the range $[-\pi / 2, \pi / 2]$, and as a result, the predicted power was dominated by the DBS component.

From these results, even if the same area is observed at same incidence angle, the variation of the moisture content of the ground and the vegetation can cause the nonphysical surface scattering component. If the vegetated terrain is composed of vertically standing trunks as modeled in Sec II-D, the incidence angle of $45^{\circ}$ is preferred to obtain stable and physically meaningful MBD results.

\section{EXPERIMENTAL VALIDATION}

In this section, we experimentally validate the theoretical description in Section II and Section III, where we have pointed out the potential incorrect prediction of the DBS component in the MBD schemes. For this purpose, a series of controlled laboratory experiments was carried out in an anechoic chamber with a simplified model of vegetated terrain. Note that the measured data presented in this section is same as those investigated in [25].

\section{A. Overview of the Experiment}

First, we describe overview of the experiment. Fig. 8 and Fig. 9 show a photograph and the measurement geometry of this experiment. According to the theoretical model presented in Section II, we modeled vegetated terrain by a cylinder and a flat plate. However, unlike the theoretical model, the experimental model had many vertical cylinders randomly distributed over the flat plate rather than a single cylinder on a plate, where all of the parts of the model were made of wood. Note that our purpose is not direct quantitative comparison between the numerical and the experimental results. Therefore, although the experimental model differs from the theoretical model, the model of the multiple cylinders was preferred because it emulates more realistic vegetated terrain and it can enhance backscattering cross-section to achieve sufficient signal-to-noise ratio in the receiver.

As discussed in Section III-C, we varied moisture contents of the model. For convenience, the large ground plate was divided into small $5 \times 5$ pieces, as shown in Fig. 9. Before carrying out a series of the experiments, the wooden plates and 


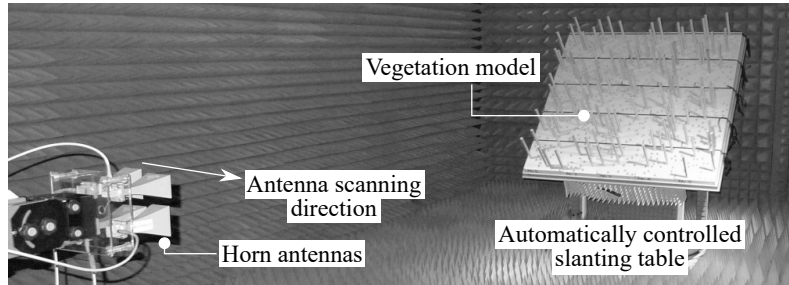

Fig. 8. A photograph of the experiment. Four standard gain horn antennas were used as the transmitting and receiving antennas. The incidence angle was continuously varied by automatically changing the slant of the table holding the vegetation model.



Fig. 9. Geometry of the experiment. Wooden cylinders vertically stand on a large $(1.5 \mathrm{~m} \times 1.5 \mathrm{~m})$ wooden plate. The locations of the cylinders were determined from uniform random numbers. Four standard gain horn antennas were connected to a VNA and scanned horizontally to create a synthetic aperture.

the cylinders were put into the water to moisten them. After removing them from the water, their weights were measured to determine the gravimetric moisture content, and then they were coated with plastic film to prevent the inside moisture from drying. In this experiment, measurement was carried out at 9 different moisture contents. We designate each wet condition by 'wet condition number,' where the number 9 indicates the wettest condition which was right after removing the model from the water, and the number 1 indicates the driest condition corresponding to the last experiment; thus, chronological order was from the wet condition number 9 to 1 .

Fig. 10 shows the variation of the gravimetric moisture contents of the ground plates and the trunk cylinders in terms of the wet condition numbers. The moisture contents were measured for 4 different pieces of the ground plate, and for 4 different cylinders. Although all the cylinders show the similar trend, however, one of the ground plate shows the significantly different moisture content compared to the other 3 plates. This was attributed to the fact that, because the plates were stacked into a water tank, a plate at a deeper location experienced a greater water pressure so that the plate absorbed more water compared to a plate at a shallower location in the water tank. Even so, we can confirm from Fig. 10 that all the water contents decreases with the wet condition number.

As depicted in Fig. 8, the coated models were mounted on a slanting table, and SAR measurements were carried

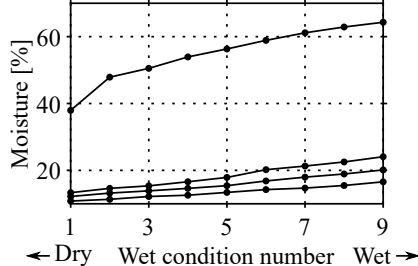

(a)

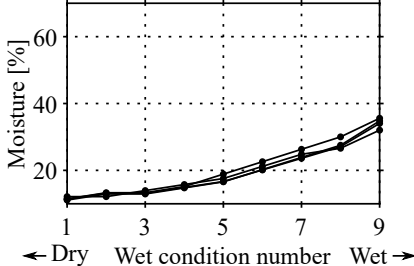

(b)
Fig. 10. Gravimetric moisture contents of the vegetation model. The moisture contents were measured at 4 different locations. (a) Ground. (b) Trunk.

TABLE III

EXPERIMENTAL PARAMETERS.

\begin{tabular}{l|l}
\hline Polarization $q p$ & $h h, h v, v h, v v$ \\
Center frequency & $10 \mathrm{GHz}$ (X-band) \\
Bandwidth & $2 \mathrm{GHz}$ \\
Frequency step & $10 \mathrm{MHz}$ \\
Incidence angle $\theta_{i}$ & $0^{\circ}$ to $90^{\circ}$ \\
Antenna distance $\rho_{0}$ & $5 \mathrm{~m}$ \\
Synthetic aperture length $2 L$ & $2 \mathrm{~m}$ \\
\hline Cylinder material & Wood \\
Cylinder diameter $a$ & $2 \mathrm{~cm}$ \\
Cylinder length $l$ & $30 \mathrm{~cm}$ \\
Cylinder orientation & Vertical \\
Cylinder density & $55.6 \mathrm{cylinders} / \mathrm{m}^{2}$ \\
\hline Plate material & Wood \\
Plate width & $1.5 \mathrm{~m}$ \\
Plate height & $1.5 \mathrm{~m}$ \\
Plate thickness & $3 \mathrm{~cm}$ \\
Moisture condition & 9 types \\
\hline
\end{tabular}

out at various incidence angles. The slant of the table were automatically controlled such that one can achieve the desired incidence angles with respect to the ground plate. Four standard gain horn antennas (Narda Model 640), which cover frequency band from $8.2 \mathrm{GHz}$ to $12.4 \mathrm{GHz}$, were used as the transmitting and receiving antennas. As shown in Fig. 9, these antennas were connected to a vector network analyzer (VNA, Keysight N5242A), which was used as a transmitter and receiver, through microwave coaxial cables; 30-dB low noise amplifiers were inserted in the receiving paths. At each incidence angle, the antennas were scanned horizontally (i.e., $y$-direction in Fig. 9) to create a synthetic aperture. By this experimental setup, polarimetric SAR measurements in the controlled conditions were extensively carried out in various combinations of the incidence angle and the moisture content.

Table III lists the experimental parameters. The center frequency was $10 \mathrm{GHz}$, and the bandwidth was $2 \mathrm{GHz}$. As depicted in Fig. 9, the distance from the antenna track to the center of the ground plate was $5 \mathrm{~m}$, and the synthetic aperture length was $2 \mathrm{~m}$. The diameter and the length of the wooden cylinder were $2 \mathrm{~cm}$ and $30 \mathrm{~cm}$, respectively. The width and height of the wooden plate were both $1.5 \mathrm{~m}$, and the thickness was $3 \mathrm{~cm}$. The total number of the cylinders was 125 , resulting the spatial density of 55.6 cylinders $/ \mathrm{m}^{2}$. The locations of these cylinders were determined from uniform random numbers.

\section{B. SAR Imaging and Interpretation}

Next, we describe SAR imaging from the measured signals and interpretation of the reconstructed spatial images. As 
shown in Fig. 9, the antennas were moved along the linear track of which the radial distance from the origin was fixed at $\rho_{0}=5 \mathrm{~m}$, and the incidence angle $\theta_{i}$ was varied by changing the slant of the vegetation model. We denote the antenna location on the linear track as $\boldsymbol{r}_{0}(u)$, where $u \in[-L, L]$ with $L=1 \mathrm{~m}$ represents the antenna location on the track and $2 L$ is the synthetic aperture length. The antenna location $\boldsymbol{r}_{0}(u)$ is expressed as follows:

$$
\boldsymbol{r}_{0}(u)=\left(\rho_{0} \sin \theta_{i}, u, \rho_{0} \cos \theta_{i}\right)
$$

We also denote an arbitrary 3-D spatial point as $\boldsymbol{r}=(x, y, z)$, and define the $q p$-polarized reflectivity at that point as $f_{q p}(\boldsymbol{r})$. Based on these notations, the $q p$-polarized scattered signal received by the antenna can be modeled by

$$
s_{q p}\left(k, \boldsymbol{r}_{0}\right)=\int_{\Omega} f_{q p}(\boldsymbol{r}) \frac{e^{-j 2 k\left|\boldsymbol{r}_{0}-\boldsymbol{r}\right|}}{\left|\boldsymbol{r}_{0}-\boldsymbol{r}\right|^{2}} d \boldsymbol{r}
$$

where $\Omega$ represents the spatial domain of the integration.

The $q p$-polarized spatial image $\psi_{q p}(\boldsymbol{r})$ was reconstructed by the following expression:

$$
\psi_{q p}(\boldsymbol{r})=\int_{0}^{\infty} \int_{-L}^{L} s_{q p}\left(k, \boldsymbol{r}_{0}\right)\left|\boldsymbol{r}_{0}-\boldsymbol{r}\right|^{2} e^{j 2 k\left|\boldsymbol{r}_{0}-\boldsymbol{r}\right|} d u d k .
$$

The simplest numerical implementation was obtained by replacing the integrals in Eq. (34) by the following summations.

$$
\begin{aligned}
\psi_{q p}\left(\boldsymbol{r}_{i}\right) \simeq \sum_{\ell} \sum_{n} s_{q p}\left[k_{\ell}, \boldsymbol{r}_{0}\left(u_{n}\right)\right] \\
\cdot\left|\boldsymbol{r}_{0}\left(u_{n}\right)-\boldsymbol{r}_{i}\right|^{2} e^{j 2 k_{\ell}\left|\boldsymbol{r}_{0}\left(u_{n}\right)-\boldsymbol{r}_{i}\right|}
\end{aligned}
$$

where $\boldsymbol{r}_{i}, k_{\ell}$, and $u_{n}$ are the discretized values of $\boldsymbol{r}, k$, and $u$, respectively, and $i, \ell, n \in\{1,2, \ldots\}$ are the indices to represent individual samples.

We constructed the scattering matrix at the image location $\boldsymbol{r}_{i}$ from the reconstructed polarimetric images $\psi_{q p}\left(\boldsymbol{r}_{i}\right)$ as

$$
\boldsymbol{S}_{m}\left(\boldsymbol{r}_{i}\right)=\left[\begin{array}{cc}
S_{h h}^{m} & S_{h v}^{m} \\
S_{v h}^{m} & S_{v v}^{m}
\end{array}\right]=\left[\begin{array}{ll}
\psi_{h h}\left(\boldsymbol{r}_{i}\right) & \psi_{h v}\left(\boldsymbol{r}_{i}\right) \\
\psi_{v h}\left(\boldsymbol{r}_{i}\right) & \psi_{v v}\left(\boldsymbol{r}_{i}\right)
\end{array}\right]
$$

where the letter $m$ indicates the measured scattering matrix. The corresponding covariance matrix $\boldsymbol{C}_{m}\left(\boldsymbol{r}_{i}\right)$ can be obtained from Eq. (3) with $\xi \rightarrow m$. Moreover, we computed the averaged covariance matrix $\left\langle\boldsymbol{C}_{m}\right\rangle$ as

$$
\left\langle\boldsymbol{C}_{m}\right\rangle=\frac{1}{N} \sum_{i} \boldsymbol{C}_{m}\left(\boldsymbol{r}_{i}\right)
$$

where the average was taken over the area that contains the backscattering from the vegetation model, and $N$ is the total number of pixels within this area.

Based on the averaged covariance matrix defined in Eq. (37), we derived the backscattering coefficients $\sigma_{q p s r}^{m}$ and the CPD $\phi_{h h v v}^{m}$, and applied the FDD to the measured averaged covariance matrix. Note that, as in the numerical simulation, we also tested the Y40 [10] and the AMBD [3], [15], and confirmed that the similar results were obtained.

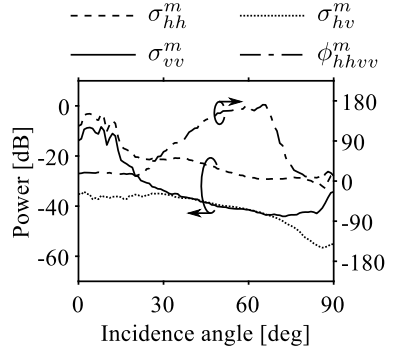

(a)

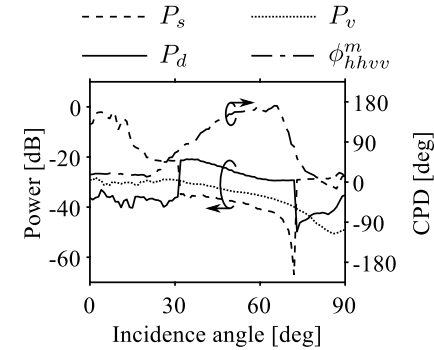

(b)
Fig. 11. Co-polarized backscattering coefficients, CPD, and FDD results (experiment, wet condition 2).

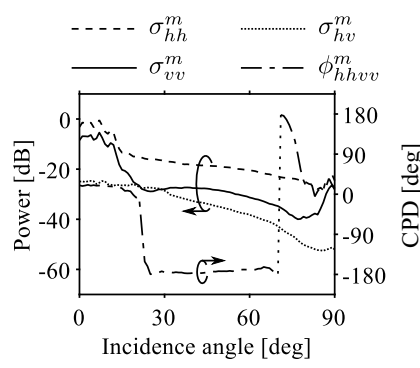

(a)

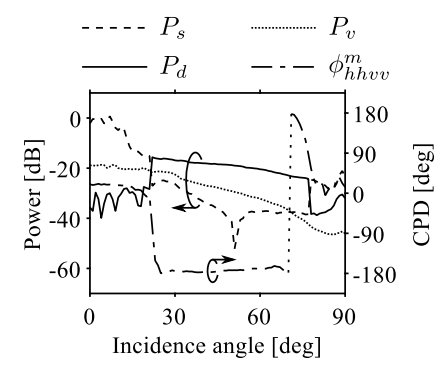

(b)
Fig. 12. Co-polarized backscattering coefficients, CPD, and FDD results (experiment, wet condition 8).

\section{Results and Discussion}

Fig. 11 and Fig. 12 show the measured backscattering coefficients and the decomposed powers using the FDD for the wet conditions 2 and 8 , respectively, plotted with the measured CPD $\phi_{h h v v}^{m}$. Note that in Fig. 11(b) and Fig. 12(b), $P_{s}=\left|f_{s}\right|^{2}$ (surface scattering), $P_{d}=\left|f_{d}\right|^{2}$ (DBS), and $P_{v}=\left|f_{v}\right|^{2}$ (volume scattering).

In Fig. 11(a), although the Brewster angle dip is unrecognizable, however, transition in the CPD indicates the existence of the two Brewster angles corresponding to the ground and the cylinders. As in the numerical model discussed in Section II-D, the $h h$-polarized backscattering coefficients are greater than the $v v$-polarized coefficients for all the incidence angles. As shown in Fig. 11(b), the DBS power is dominant when the CPD is greater than $90^{\circ}$. However, the decomposed power is dominated by the surface component when the CPD is smaller than $90^{\circ}$, which is physically incorrect result. Similar trend can be observed in the wet condition 8 presented in Fig. 12, where we can recognize the two Brewster angle dips at the incidence angles around $25^{\circ}$ and $75^{\circ}$ in Fig. 12(a). In Fig. 12(b), the DBS stays to be dominant for wider angular range compared to Fig. 11(b), because the range between the two Brewster angles is wider because of the larger moisture content.

Fig. 13(a) and (b) show the decomposed power using the FDD with respect to the wet condition numbers at the incidence angle $45^{\circ}$ and $30^{\circ}$, respectively. From Fig. 13(a), we can see that the DBS dominates for all the wet conditions at the incidence angle of $45^{\circ}$, as expected. However, if the incidence angle was changed to $30^{\circ}$, the DBS power is dominant only from the wet condition numbers 5 to 9 . For the wet condition numbers 1 to 4 , the surface contribution is estimated to be 


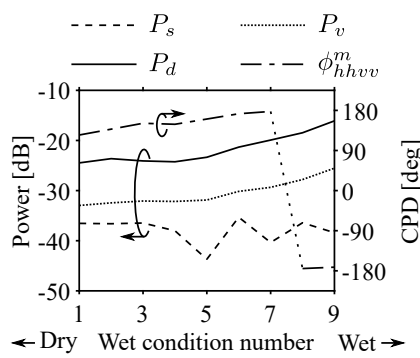

(a)



(b)
Fig. 13. CPD and FDD results with respect to the wet condition number (experiment). (a) $\theta_{i}=45^{\circ}$. (b) $\theta_{i}=30^{\circ}$.

dominant. From these experimental results, we can confirm that the problem of incorrect DBS power estimation certainly occurred in the measured data.

\section{CONCLUSiON}

In this research, we discuss a potential problem of the model-based polarimetric decomposition algorithms that the expected double-bounce component can be incorrectly estimated as the surface scattering component, owing to the phase transition of the Brewster angles corresponding to the ground and the vegetation. We explain this phenomenon in detail by a simple numerical backscattering model for vegetated terrain, and the theoretical results are confirmed by the series of controlled experiments carried out in an anechoic chamber. The results show that care must be taken when interpreting the decomposed results of the vegetated terrain. If the vegetated terrain is composed of vertically standing trunks on the ground, the incidence angle around $45^{\circ}$ is preferred to obtain stable and physically meaningful decomposed results.

\section{REFERENCES}

[1] S. Cloude, Polarisation: Applications in Remote Sensing, Illustrated edition. Oxford; New York: Oxford University Press, 2009.

[2] J.-S. Lee and E. Pottier, Polarimetric Radar Imaging: From Basics to Applications, 1st edition. Boca Raton: CRC Press, 2009.

[3] J. J. van Zyl, Synthetic Aperture Radar Polarimetry, 1st edition. Hoboken, NJ: Wiley, 2011

[4] Y. Yamaguchi, Polarimetric SAR Imaging: Theory and Applications, 1st edition. Boca Raton: CRC Press, 2020.

[5] J. J. van Zyl, "Application of Cloude's target decomposition theorem to polarimetric imaging radar data," in Radar Polarimetry, Feb. 1993, vol. 1748, pp. 184-191.

[6] S. R. Cloude and E. Pottier, "A review of target decomposition theorems in radar polarimetry," IEEE Trans. Geosci. Remote Sens., vol. 34, no. 2, pp. 498-518, Mar. 1996.

[7] S. R. Cloude and E. Pottier, "An entropy based classification scheme for land applications of polarimetric SAR," IEEE Trans. Geosci. Remote Sens., vol. 35, no. 1, pp. 68-78, Jan. 1997.

[8] R. Touzi, "Target Scattering Decomposition in Terms of Roll-Invariant Target Parameters," IEEE Trans. Geosci. Remote Sens., vol. 45, no. 1, pp. 73-84, Jan. 2007.

[9] A. Freeman and S. L. Durden, "A three-component scattering model for polarimetric SAR data," IEEE Trans. Geosci. Remote Sens., vol. 36, no. 3, pp. 963-973, May 1998.

[10] Y. Yamaguchi, T. Moriyama, M. Ishido, and H. Yamada, "Fourcomponent scattering model for polarimetric SAR image decomposition," IEEE Trans. Geosci. Remote Sens., vol. 43, no. 8, pp. 1699-1706, Aug. 2005.

[11] A. Freeman, "Fitting a Two-Component Scattering Model to Polarimetric SAR Data From Forests," IEEE Trans. Geosci. Remote Sens., vol. 45, no. 8 , pp. 2583-2592, Aug. 2007
[12] Y. Yajima, Y. Yamaguchi, R. Sato, H. Yamada, and W.-M. Boerner, "POLSAR Image Analysis of Wetlands Using a Modified FourComponent Scattering Power Decomposition," IEEE Trans. Geosci. Remote Sens., vol. 46, no. 6, pp. 1667-1673, Jun. 2008.

[13] W. An, Y. Cui, and J. Yang, "Three-Component Model-Based Decomposition for Polarimetric SAR Data," IEEE Trans. Geosci. Remote Sens., vol. 48, no. 6, pp. 2732-2739, Jun. 2010.

[14] M. Arii, J. J. van Zyl, and Y. Kim, "A General Characterization for Polarimetric Scattering From Vegetation Canopies," IEEE Trans. Geosci. Remote Sens., vol. 48, no. 9, pp. 3349-3357, Sep. 2010.

[15] M. Arii, J. J. van Zyl, and Y. Kim, "Adaptive Model-Based Decomposition of Polarimetric SAR Covariance Matrices," IEEE Trans. Geosci. Remote Sens., vol. 49, no. 3, pp. 1104-1113, Mar. 2011.

[16] Y. Yamaguchi, A. Sato, W.-M. Boerner, R. Sato, and H. Yamada, "FourComponent Scattering Power Decomposition With Rotation of Coherency Matrix," IEEE Trans. Geosci. Remote Sens., vol. 49, no. 6, pp. 2251-2258, Jun. 2011.

[17] J. J. van Zyl, M. Arii, and Y. Kim, "Model-Based Decomposition of Polarimetric SAR Covariance Matrices Constrained for Nonnegative Eigenvalues," IEEE Trans. Geosci. Remote Sens., vol. 49, no. 9, pp. $3452-$ 3459, Sep. 2011.

[18] S.-W. Chen, M. Ohki, M. Shimada, and M. Sato, "Deorientation Effect Investigation for Model-Based Decomposition Over Oriented Built-Up Areas," IEEE Geosci. Remote Sens. Lett., vol. 10, no. 2, pp. 273-277, Mar. 2013.

[19] S.-W. Chen, X.-S. Wang, S.-P. Xiao, and M. Sato, "General Polarimetric Model-Based Decomposition for Coherency Matrix," IEEE Trans. Geosci. Remote Sens., vol. 52, no. 3, pp. 1843-1855, Mar. 2014.

[20] Y. Cui, Y. Yamaguchi, J. Yang, H. Kobayashi, S.-E. Park, and G. Singh, "On Complete Model-Based Decomposition of Polarimetric SAR Coherency Matrix Data," IEEE Trans. Geosci. Remote Sens., vol. 52, no. 4, pp. 1991-2001, Apr. 2014.

[21] J.-S. Lee, T. L. Ainsworth, and Y. Wang, "Generalized Polarimetric Model-Based Decompositions Using Incoherent Scattering Models," IEEE Trans. Geosci. Remote Sens., vol. 52, no. 5, pp. 2474-2491, May 2014.

[22] G. Singh and Y. Yamaguchi, "Model-Based Six-Component Scattering Matrix Power Decomposition," IEEE Trans. Geosci. Remote Sens., vol. 56, no. 10 , pp. 5687-5704, Oct. 2018

[23] M. T. Hallikainen, F. T. Ulaby, M. C. Dobson, M. A. El-rayes, and L. Wu, "Microwave Dielectric Behavior of Wet Soil-Part 1: Empirical Models and Experimental Observations," IEEE Trans. Geosci. Remote Sens., vol. GE-23, no. 1, pp. 25-34, Jan. 1985.

[24] F. T. Ulaby and M. A. El-rayes, "Microwave Dielectric Spectrum of Vegetation-Part II: Dual-Dispersion Model," IEEE Trans. Geosci. Remote Sens., vol. GE-25, no. 5, pp. 550-557, Sep. 1987.

[25] T. Watanabe, H. Yamada, M. Arii, R. Sato, S.-E. Park, and Y. Yamaguchi, "Study on Moisture Effects on Polarimetric Radar Backscatter from Forested Terrain,"

[26] K. Ouchi and C.-S. Yang, "Brewster Angle Damping Observed in the TerraSAR-X Synthetic Aperture Radar Images of Man-Made Targets," IEEE Geosci. Remote Sens. Lett., vol. 15, no. 4, pp. 532-536, Apr. 2018. IEICE Trans. Commun., vol. E97.B, no. 10, pp. 2074-2082, 2014.

[27] S. L. Durden, J. J. van Zyl, and H. A. Zebker, "Modeling and observation of the radar polarization signature of forested areas," IEEE Trans. Geosci. Remote Sens., vol. 27, no. 3, pp. 290-301, May 1989.

[28] K. Sarabandi, "Electromagnetic Scattering from Vegetation Canopies," $\mathrm{PhD}$ dissertation in The University of Michigan, 1989.

[29] F. T. Ulaby and C. Elachi, Eds., Radar Polarimetry for Geoscience Applications, Illustrated edition. Norwood, MA: Artech House Publishers, 1990.

[30] J. J. van Zyl, "The effect of topography on radar scattering from vegetated areas," IEEE Trans. Geosci. Remote Sens., vol. 31, no. 1, pp. 153-160, Jan. 1993.

[31] A. Ishimaru, Wave Propagation and Scattering in Random Media, WileyIEEE Press, 1997. 


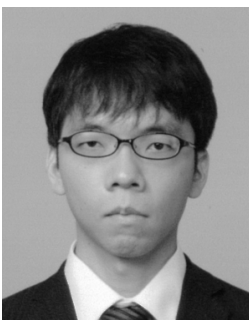

Takuma Watanabe (M'12) received the B.E., M.E., and Dr.Eng. degrees in electrical and information engineering from Niigata University, Niigata, Japan, in 2010,2012 , and 2015, respectively.

From 2015 to 2017, he was with the Central Research Institute of Electric Power Industry, Yokosuka, Japan. From 2017 to 2018, he was with PASCO Corporation, Tokyo, Japan. Since 2018, he has been with Fujitsu Ltd., Kawasaki, Japan, where he is currently involved in the development of RCS evaluation system for fighter aircrafts. His research interests include image reconstruction problems, SAR signal processing, array signal processing, and electromagnetic scattering problems.

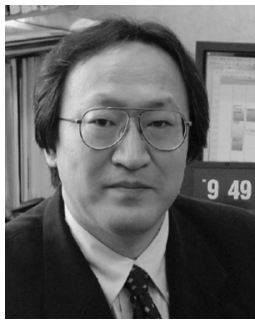

Hiroyoshi Yamada (M'93) received the B.E., M.E., and Dr.Eng. degrees in electronic engineering from Hokkaido University, Sapporo, Japan, in 1988, 1990, and 1993, respectively.

Since 1993, he has been with the Faculty of Engineering, Niigata University, Niigata, Japan, where he is currently a Professor. From 2000 to 2001, he was a Visiting Scientist with the Jet Propulsion Laboratory, California Institute of Technology, Pasadena, CA, USA. His research interests include array signal high-resolution techniques. processing, polarimetric radar interferometry, and 